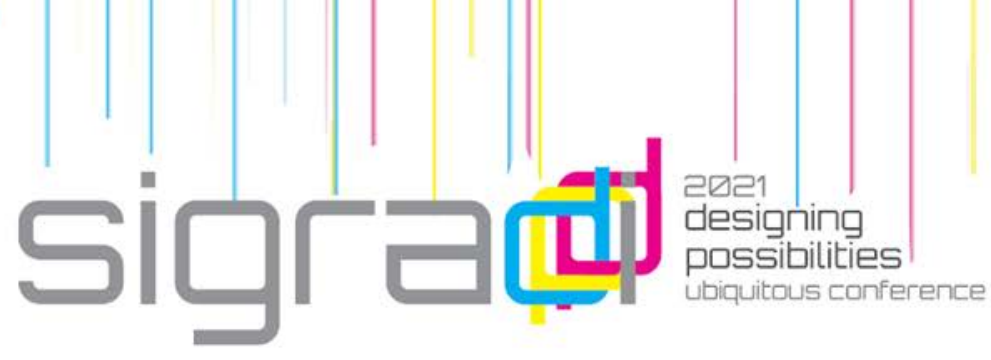

\title{
Parametric Urbanism: Multi-Criteria Optimization for a Sustainable Neighborhood in São José Dos Campos
}

\author{
Adriane Eloah ${ }^{1}$, Natália Queiroz ${ }^{2}$, Leonardo Coelho² \\ ${ }^{1}$ Centro Universitário Belas Artes de São Paulo, Brasil \\ adrianeeloah@gmail.com \\ ${ }^{2}$ Universidade Federal de Santa Catarina, Brasil \\ nataliaqueiroz@labcon.ufsc.br \\ leonardo.coelho@belasartes.br
}

\begin{abstract}
Based on the concepts of Sustainable Urbanism and performance-based design, this work proposes the development of a neighborhood in the city of São José dos Campos (SJC), São Paulo-Brazil. The performance-based design method was used through parametric algorithmic tools - such as Grasshopper - for construction, analysis and the urban design multicriteria optimization. Five optimization functions were used: walkability, sunset view, radiation in hot periods, minimum hours of sun exposition and maximum number of floors. The use of optimization software accommodates the numerous conflicting requirements of the model. The results obtained are a systematization of the process and a system that allows various urban solutions based on numerical performance criteria. The selected solutions achieved walkability indicators greater than $80 \%$.
\end{abstract}

Keywords: Parametric urban design, Multi-objective optimization, Performance-based design, Sustainable design, Walkability

\section{Introdução}

Este artigo apresenta o projeto de um bairro segundo diretrizes de Urbanismo Sustentável para a cidade de São José dos Campos (SJC). Para tal, explora o conceito de projeto baseado em desempenho através de modelagem paramétrica e otimização. Para a melhor compreensão do artigo, serão apresentados inicialmente três conceitos chave para o desenvolvimento da pesquisa: urbanismo sustentável, modelos de parametrização para projetos 
urbanísticos e projetos guiado por desempenho. Em seguida, será caracterizado o município de SJC, recorte de estudo deste trabalho.

\subsection{Projeto guiado pelo desempenho}

Muitos termos são utilizados para definir projeto guiado pelo desempenho: peformance-based-design, peformalism, peformative architecture (Grobman \& Neuman, 2008; Kolarevic, 2004; Oxman, 2017). Esses termos designam o processo de projeto onde os indicadores de desempenho são os guias-chave da geração da geometria. Nela, a equipe projetual pode obter retornos mais rápidos sobre o desempenho da solução e ser guiada por um processo de otimização que executa loops de análises associados ao problema de projeto (Evelin \& Jason, 2014; Nguyen, et al., 2014).

A problemática de projeto possui característica multidisciplinar, de natureza quantitativa e qualitativa. Por isso, a literatura vem recomendando a utilização de modelos de otimização multicritério em detrimento do unicritério (Kirimtat, et al., 2019; Zhao \& de Angelis, 2019). O modelo multicritério representa o problema com múltiplas funções. Assim, não existe uma solução determinada como a melhor, mas um conjunto de soluções otimizadas em um universo de possibilidades de forma a caracterizar o problema. Os projetistas definem soluções de interesse. Em contraponto, a vantagem em possibilitar múltiplas funções também pode caracterizar desvantagem. A complexidade pode gerar problemas difíceis de caracterizar, podem consumir tempo ou o número de variações pode impossibilitar a execução (Wortmann \& Nannicini, 2017).

O conceito de desempenho aqui empregado extrapola a abordagem física ou técnica, intencionando ter interpretação holística que pode incorporar aspectos financeiros, espaciais, sociais e culturais, desde que sejam quantificáveis. Pesquisas nesta área buscam processos digitais que combinam diferentes abordagens integradas ao modelo 3D e aspiram soluções para o desenvolvimento sustentável (Kolarevic \& Malkawi, 2005).

Os processos de otimização permitem a combinação da geração da forma e a negociação do universo de soluções possíveis com princípios de desempenho (Anton \& Tănase, 2016). Como avanço tem-se a possibilidade da exploração "exaustiva" do espaço do problema, possibilitando a exploração de objetivos de projeto, da escolha de soluções, do estabelecimento de geometrias customizadas e da realização de análises estatísticas (Aish, 2011).

\subsection{Urbanismo Sustentável}

A população brasileira é majoritariamente urbana, correspondendo atualmente a mais de $85 \%$ do total (IBGE, 2017). Globalmente o fenômeno é também relevante, pois mais de $50 \%$ da população mundial vive em cidades e até 2050 esse valor atingirá mais de 75\% (Leite, 2012). 
Embora as cidades tenham se tornado potências de desenvolvimento econômico e social do século XXI (Rodriguez, 2016), a popularização do transporte motorizado no século XX promoveu espraiamento urbano e bairros monofuncionais residenciais ligados aos centros urbanos por vias de fluxo rápido, produzindo assim cidades dormitórios distantes de trabalhos, comércios e serviços (Macedo, 2007). O crescimento urbano tem produzido cidades inseguras e de difícil mobilidade (Rodriguez, 2016). Desde o final do século XX práticas de sustentabilidade têm sido incorporadas em projetos urbanísticos para mitigar impactos decorrentes da sua implantação, sendo exemplo nacional e internacional, respectivamente, o bairro da Pedra Branca em Palhoça - SC (2013) e a cidade de Masdar nos Emirados Árabes (2010).

O Urbanismo Sustentável surge com a ideia de redesenhar ambientes construídos de maneira a obter maior qualidade de vida e um estilo de vida saudável e sustentável (Farr, 2013). Ele deriva de três movimentos de reforma urbana do final do século XX: o crescimento urbano inteligente, o Novo Urbanismo e as construções sustentáveis. Esses movimentos, por si só, não são capazes de alcançar seus objetivos. Por isso, considera-se importante unilos e entrelaçá-los em uma filosofia de desenho urbano que crie ambientes humanos mais sustentáveis (Farr, 2013).

O conceito contempla comunidades densas com limites e centros definidos, bom sistema de transporte público, diversidade de uso do solo e tipologias de edifícios, atrativos para mobilidade ativa, acesso humano à natureza, eficiência energética, melhor uso das águas e conexões verdes (biofilia) (Farr, 2013; Leite, 2012; Rodriguez, 2016). A demanda viária e espraiamento urbano devem ser reduzidos por meio de cidades mais densas e compactas. Assim, minimiza-se a dependência do automóvel (Leite, 2012; Rodriguez, 2016).

\subsection{Modelos de parametrização para projetos urbanísticos}

A medida de baixo impacto para cidades e bairros impõe a busca por técnicas para otimizar projetos e operações (Leite, 2012). Considerando as dificuldades de utilizar multivariáveis e mapeamento de dados, as ferramentas digitais de modelagem evoluíram de maneira a colaborar na utilização de dados e informação da construção. Elas são aliadas para soluções urbanas complexas (Carone, 2016). Nesse sentido, as ferramentas paramétricas, por exemplo, estão em crescimento no campo do urbanismo, porém ainda em menor número que proposições na escala de arquitetura e design (Lima, et al., 2020).

Para mensurar e analisar métricas do Desenvolvimento Orientado pelo Transporte (DOT), Lima (2019) desenvolveu o CityMetrics - modelo para atuar dentro da ferramenta de linguagem de programação visual (Lima, et al, 2020). Trata-se de um conjunto de ferramentas algorítmicas - algumas, desenvolvidas do zero e outras adaptadas - que fazem uso de métricas de avaliação de 
desempenho para criar regras e operações lógicas (Lima, 2019). Ele permite análises de caminhabilidade com índices do DOT, mensurando distâncias físicas e topológicas, bem como permite a análise de densidade com indicadores do Spacematrix. Esses resultados podem ser associados à otimização multicritério e unicritério (Lima, 2019).

No mesmo contexto, Cichocka (2015) faz a otimização de um projeto urbano generativo para promover a caminhabilidade de um bairro que está sujeito a aumentar sua densidade. A autora utiliza os softwares Rhinoceros/ Grasshopper com o plugin ShortestWalk para analisar o walkscore ${ }^{1}$ em função da distância das amenidades existentes. Em seguida, a autora utiliza o Galápagos para estabelecer localização aleatória das amenidades novas na malha viária. O resultado foi o aumento do walkscore em 25 pontos.

Para aferir qual melhor tipologia de tecido urbano para caminhabilidade, Lima et al. (2021) concluíram que o uso do voronoi de polígonos irregulares ou seja: formado por pontos de distâncias diferentes - em detrimento do grid convencional, apresenta maior índice de caminhabilidade, computado através das distâncias entre residências e diversidade de usos.

\section{Caracterização do recorte de estudo}

SJC está na Região Metropolitana do Vale do Paraíba (RMVP), Latitude: $23^{\circ}$ 10' 45" Sul, incluída na Macrometrópole Paulista. Possui 1.099,41 Km² e 710.654 mil habitantes (Seade, 2021). A localização próxima à Rodovia Presidente Dutra contribuiu para a instalação de indústrias, universidades e centros de pesquisas. Por essa razão, caracteriza-se por ser um polo tecnológico, industrial e de serviços da RMVP (IPPLAN, 2014).

A cidade é esparsa em decorrência das dificuldades de integração da malha urbana (PDDI, 2018). Essas dificuldades ocorreram por diversos fatores: barreiras físicas, desenho dos parcelamentos do solo, formação de núcleos urbanos de acesso controlado, malha viária incipiente e presença de vazios urbanos em meio a áreas providas de boa rede de infraestrutura. O município extrapola a média de uso de automóveis para cidades de porte similar no Brasil (IPPLAN, 2014), sendo eles elementos estruturadores da mobilidade da população. Seu uso tem crescido e tornando o sistema viário saturado, motivando interesse por parte do poder público em estabelecer "centralidades polarizadoras" no município (Moura, 2006; PDDI, 2018).

\footnotetext{
${ }^{1}$ Pontuação de 0 a 100 em função da máxima transitabilidade do bairro com o mínimo de distância possível
} 


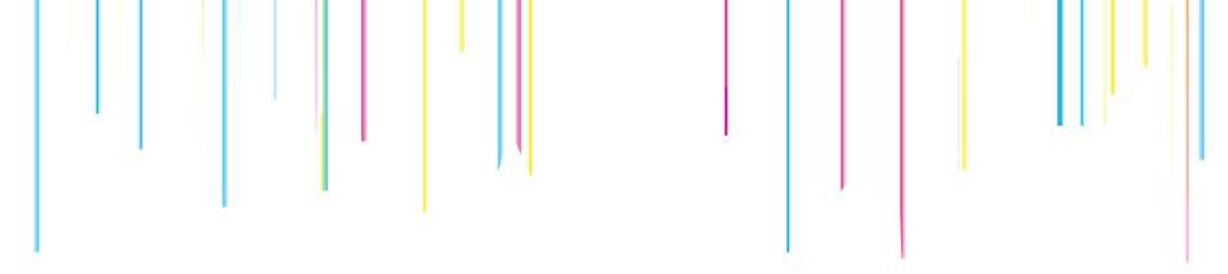

\section{Metodologia}

O trabalho usa métodos quantitativos e qualitativos. Usa ferramentas generativas para projeto urbano com princípios de sustentabilidade. Explora conceitos como caminhabilidade, a diversidade do uso do solo, alto desempenho dos edifícios e biofilia (Farr, 2013). Utiliza-se o método de projeto baseado em desempenho (Oxman, 2008) por meio de ferramentas algorítmicoparamétricas para a construção, análise e otimização multicritério.

Inicialmente, realizou-se levantamento bibliográfico do acervo da Prefeitura de SJC bem como livros e artigos de revista. As etapas práticas foram divididas em 4 partes: i) levantamento de dados; ii) parametrização do modelo urbano; iii) análise do modelo; iv) otimização multicritério.

\subsection{Levantamento de dados}

Inicialmente, elencaram-se dados urbanos e gleba. Foram inventariados estudos prévios e dados urbanísticos, demográficos e socioeconômicos e a legislação urbana e ambiental. A gleba é um vazio em meio urbano e ocorrem dinâmicas rurais e pecuaristas. As atividades trazem valor afetivo. A população utiliza o local para assistir o pôr-do-sol e os animais. O bairro Aquarius concentra famílias de classe média alta e alta, com faixa etária predominante entre 30 e 59 anos (IPPAN, 2014). A região é constituída por áreas urbanas periféricas, e presença de condomínios, barreiras físicas, e acessibilidade precária (figura 1).

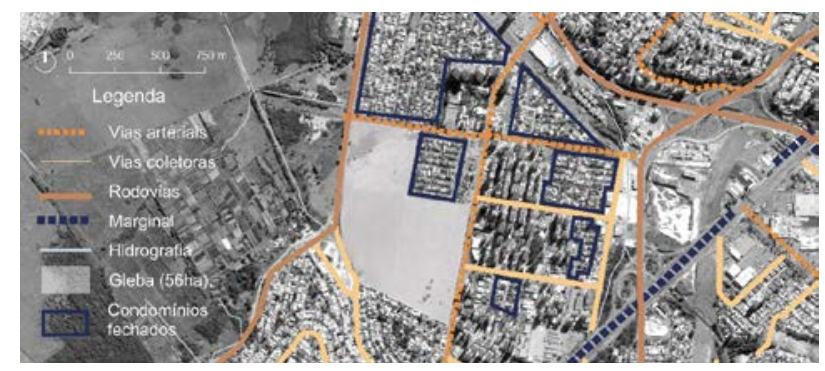

Figura 1. Gleba selecionada, cor da gleba na legenda. Fonte: Elaborado pelos autores com base no Bing Satélite, 2021

O estudo morfológico utilizou definição de Queiroz (2020) para declividade e análise de escoamento. O local é plano em sua maior parte (declive menor que $3 \%$ ) e possui um trecho com declive mais acentuado de até $10 \%$ onde havia uma nascente e coincide com o escoamento da área. O critério selecionou um parque fixo para drenagem. Na sequência, houve os seguintes procedimentos:

1. Levantamento da hierarquia viária;

2. Levantamento de projeto da prefeitura para região; 
3. Visita local para mapeamento, através da proposta de Lynch (2011);

4. Levantamento das amenidades existentes em um raio de $1,5 \mathrm{KM}$;

5. Inserção das informações levantadas no software Rhinoceros 3D.

\subsection{Parametrização do modelo urbano}

O software escolhido para a modelagem urbana foi o Rhinoceros $3 D$ e o Grasshopper, pois permitem a execução de múltiplas alternativas de projeto a partir dos parâmetros urbanos. A figura 2 mostra o diagrama resumido do modelo paramétrico desenvolvido. Os parâmetros variáveis do modelo são:

1. largura definida de acordo com cada hierarquia viária;

2. parcelamento de superquadras usando diagrama de voronoi;

3. Parcelamento das quadras com áreas idênticas pré-definidas utilizando o algoritmo Divide by Area (Dennemark, 2018).

4. modelo de parque linear do pôr do sol com largura variável,

5. Parcelamento dos lotes, seguindo dois modelos: parcelamento dos lotes em áreas idênticas (quadras menores), e modelo com parcelamento periférico, com praça no centro da quadra, inspirado no modelo Citadel Block e no Patio block (A+T, n.d.). Utilizou-se o plugin Decoding Spaces.

6. Construção dos edifícios de acordo com distribuição de densidade prédefinida de $1209 \mathrm{hab} / \mathrm{ha}$ (calculado a partir dos indicadores máximos de ocupação pela prefeitura). A distribuição da densidade foi associada a aproximação com as vias e proporcional à hierarquia viária. Os edifícios obedecem a indicadores urbanos de aproveitamento do solo calculados por quadra. O modelo permite distribuição heterogênea ou homogênea, sendo que a versão heterogênea segue uma progressão aritmética que varia a área construída por lote de forma a gerar rugosidade.

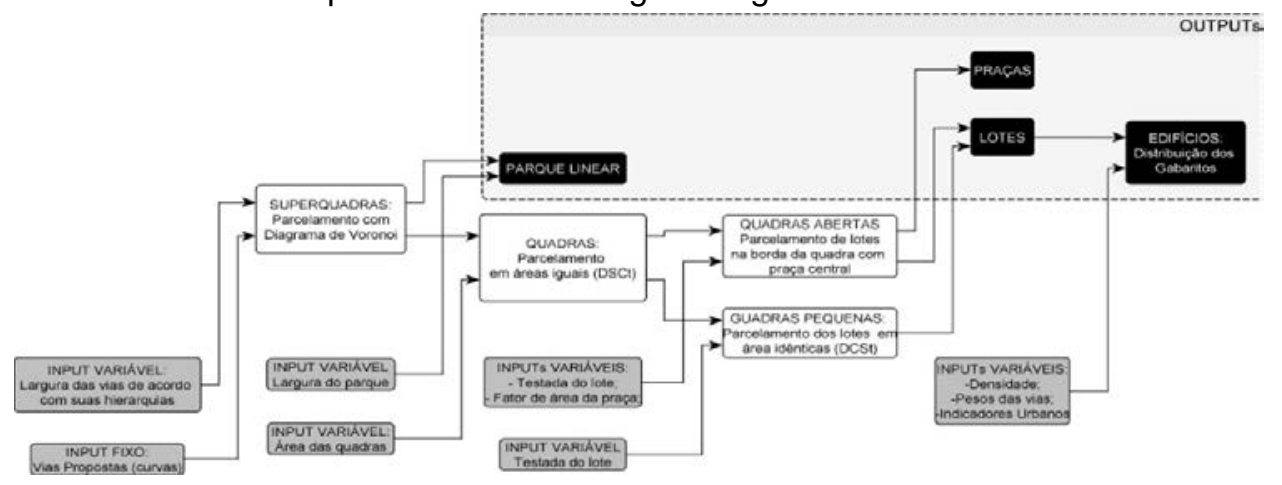

Figura 2. Diagrama com fluxograma de inputs e outputs aplicados no Grasshopper. Fonte: Autores, 2021 


\subsection{Análise do modelo}

O modelo urbano foi integrado ao modelo analítico para cálculo dos indicadores de desempenho. Quatro análises foram integradas ao modelo paramétrico: caminhabilidade; dois indicadores simplificados de conforto dos edifícios e vista do pôr do sol. A análise de caminhabilidade considerou o levantamento inicial dos usos do solo e utilizou a definição de Cichocka (2015). As amenidades foram definidas como: alimentação, serviços e comércios, educação, áreas livres e saúde. A caminhabilidade do bairro proposto foi avaliada através do cálculo de walkscore, para cada edifício (tabela 1, equação 1).

A distância de $366 \mathrm{~m}$ é percorrida a pé em 5 minutos e a de $2196 \mathrm{~m}$ em 30 minutos, ambos à velocidade de 1,22m/s (idades 14-65) (Cichocka, 2015). Através destes valores, foi estabelecida a distância de influência das amenidades para necessidades humanas semanais e diárias. Para a quantidade de amenidades adicionais necessárias utilizou-se o conceito da Cidade de 15 minutos, que considera o raio de caminhabilidade e a densidade necessária para manter os usos descriminados como relevantes (Duany \& Steuteville, 2021). Dezessete amenidades foram adicionadas e distribuídas pelo algoritmo de otimização em função da densidade adquirida: 5 para alimentação, 7 para serviços e comércios, 3 para educação, 2 para saúde e múltiplas áreas livres

Tabela 1. Parâmetros utilizados no cálculo de caminhabilidade.

\begin{tabular}{cccc} 
Walkscore & Descrição & $\begin{array}{c}\text { Tempo de } \\
\text { caminhada (min) }\end{array}$ & $\begin{array}{c}\text { Distância (m) } \\
\text { v= 1,22m/s }\end{array}$ \\
\hline $\mathbf{1 0 0}$ & Paraíso do pedestre & $<5$ & $<366$ \\
\hline $\mathbf{9 0 - 1 0 0}$ & Afazeres diários não necessitam de carro & $5-7,4$ & $366-549$ \\
\hline $\mathbf{7 0 - 8 9}$ & Afazeres diários não necessitam de carro & $7,5-12.4$ & $550-915$ \\
\hline $\mathbf{5 0 - 6 9}$ & Algumas amenidades dentro de distância caminhável & $12,5-17,4$ & $916-1281$ \\
\hline $\mathbf{2 5 - 4 9}$ & Poucas amenidades dentro de distância caminhável & $17,5-23,8$ & $1282-1739$ \\
\hline $\mathbf{0 - 2 4}$ & Quase todos os afazeres necessitam de carro & $23,9-30$ & $1740-2196$ \\
\hline $\mathbf{0}$ & Dependendo de carro & $>30$ & $>2196$ \\
\hline
\end{tabular}

Fonte: Elaborado pelos autores com base em Cichocka, 2015

$$
\begin{gathered}
W_{1}=100, d \in\langle 0 \mid 366\rangle \\
W_{2}=100-\frac{d-366}{18.3}, d \in(366 \mid 2196) \\
W_{3}=0, d \in\{2196 \mid+\infty\} \\
\mathrm{f}(\mathrm{d})=\mathrm{W} 1 \mathrm{n} 1+\mathrm{W} 2 \mathrm{n} 2+\mathrm{W} 3 \mathrm{n} 3 / \mathrm{n} 1+\mathrm{n} 2+\mathrm{n} 3
\end{gathered}
$$

Onde:

$f(d)$ é a função de aptidão. d é a menor distância entre os edifícios residenciais e amenidades. n1, n2 e n3 são respectivamente a soma dos edifícios com walkscore 100, entre 0 e 100 e $0 . n 1+n 2+n 3$ número total de edifícios avaliados.

A análise da vista do pôr do sol foi realizada usando múltiplas isovistas produzidas a partir de uma malha de pontos em relação a posição do sol no poente ao longo do ano (amostragem de 15 posições ao longo do ano). 0 cruzamento das isovistas, permite o cálculo do percentual de ocorrências do 
ano onde é possível visualizar o pôr do sol sem obstáculos no parque linear. Foi utilizado o plugin Honeybee para Grasshopper e a geometria solar de SJC.

O modelo de conforto computou dois indicadores: exposição da radiação solar em períodos quentes (acima de $24^{\circ} \mathrm{C}$ ) e áreas das fachadas que recebem um mínimo de $20 \%$ de horas de sol no ano. No primeiro caso, a intenção é reduzir a exposição da radiação em períodos quentes de forma a reduzir a demanda de condicionamento artificial e a produção de calor antropogênico. Em relação à área com mínimo de sol, a intenção é permitir qualidade do ar interno pela ação bactericida do sol. Para ambos se utilizou o plugin Honeybee para Grasshopper e o arquivo de extensão EPW (TMYX) de SJC.

\subsection{Otimização multicritério}

Para o modelo de otimização, utilizou-se o plug-in Octopus para Grasshopper e o algoritmo Hype por permitir otimização multicritério baseados na fronteira de pareto e ter um algoritmo de otimização recente. Cinco objetivos foram considerados. As funções objetivas otimizadas são apresentadas na tabela 2. O processo de otimização varia os seguintes parâmetros: taxa de ocupação dos edifícios, coeficiente de aproveitamento, tamanho dos terrenos (testada frontal), percentual de área da praça interna das quadras, localização dos centros das células voronoi, variação da posição das novas amenidades.

Tabela 2. Indicadores de desempenho utilizados.

\begin{tabular}{c|l} 
Caminhabilidade & $\begin{array}{l}\text { Depende da localização dos novos tipos de amenidades e das formas dos polígonos } \\
\text { gerados pelo Voronoi. A função de otimização considerou o valor médio estipulado } \\
\text { pelo walkscore de todas as edificações. }\end{array}$ \\
\hline Visibilidade do pôr do sol & $\begin{array}{l}\text { Depende da forma do parque linear, distribuição dos gabaritos em seu entorno, e da } \\
\text { relação com a posição do sol poente ao longo do ano. A função de otimização } \\
\text { considerou a média das ocorrências de vista do pôr do sol anual a uma altura de 1,60m } \\
\text { calculados para os centros de uma malha quadrangular distribuída no parque linear (\%). }\end{array}$ \\
\hline $\begin{array}{c}\text { Horas de sol durante o ano e } \\
\text { sombreamento em períodos } \\
\text { quentes }\end{array}$ & $\begin{array}{l}\text { O primeiro considerou o valor total da área de fachada que atendia este critério (m). } \\
\text { Para o sombreamento em períodos quentes foi considerado o valor total anual de } \\
\text { radiação sobre as fachadas (KW). }\end{array}$ \\
\hline $\begin{array}{c}\text { Número máximo de } \\
\text { pavimentos }\end{array}$ & $\begin{array}{l}\text { Para obter uma solução que satisfaça os critérios anteriores, mas que preserve um } \\
\text { número controlado de pavimentos este critério qualificador foi adicionado. }\end{array}$ \\
\hline \multicolumn{1}{c|}{ Fonte: Autores, 2021 }
\end{tabular}

\section{$4 \quad$ Resultados}

Um dos principais resultados do trabalho é o modelo paramétrico urbano flexível. Onde diversas soluções foram estabelecidas analiticamente e puderam ser escolhidas baseadas em desempenho focado no desenvolvimento sustentável.

Foram realizadas 18 gerações de 100 ciclos analíticos durante a otimização. Cada ciclo obteve um tempo médio de $165 \mathrm{~s}$. Após a otimização, o modelo paramétrico permitiu a seleção de centenas de soluções possíveis com base 


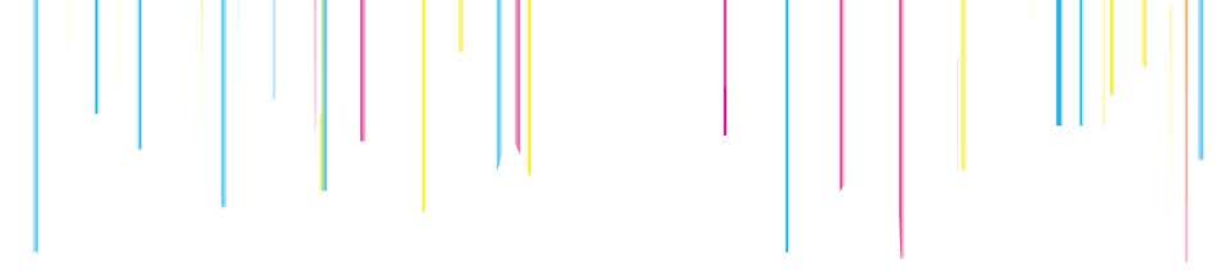

em valores analíticos conhecidos. A Figura 3 mostra os gráficos resultante da otimização multicritério. São eles, os gráficos de dispersão 3D e de coordenadas paralelas com marcação das soluções de interesse em amarelo. O critério para escolha das soluções de interesse foram as soluções com menor gabarito máximo que atendiam os melhores critérios entre as outras funções.

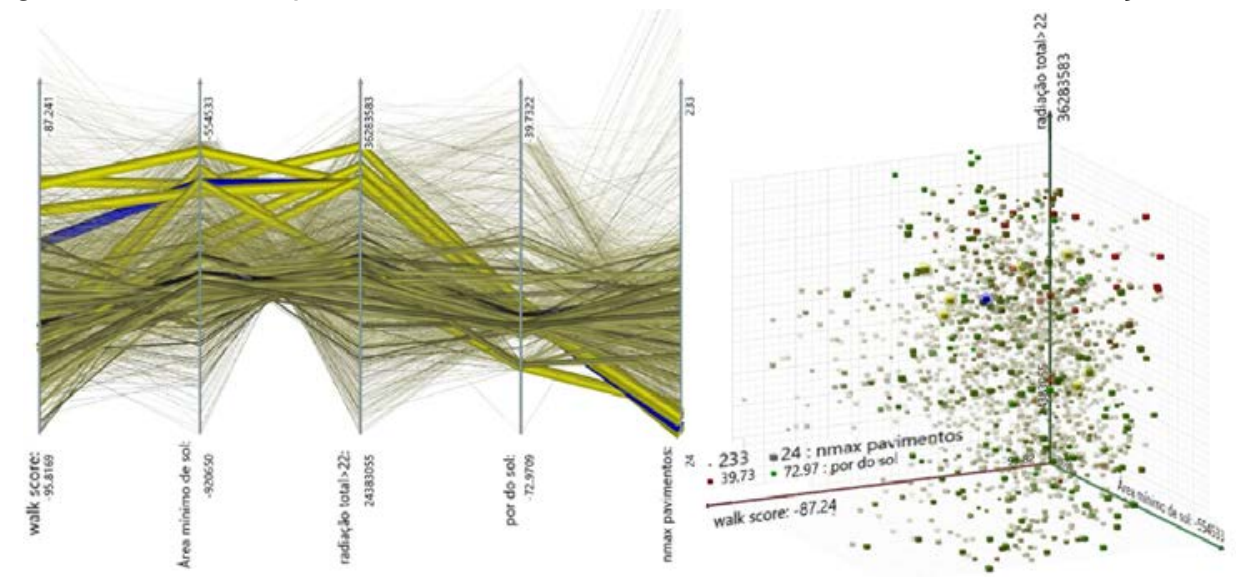

Figura 3. Gráfico de coordenadas paralelas e dispersão 3d da otimização. Fonte: Autores, 2021

A tabela 3 e a figura 4 mostram 8 e 6 soluções de interesse respectivamente. As soluções que obtiveram bons resultados para atendimento do mínimo de horas de sol (menor auto sombreamento excessivo nas fachadas) produziam maior variação do número de gabaritos (rugosidade). Inclusive há soluções que apresentam lotes vazios. Consequentemente, em conjunto com a alta densidade aplicada, houve ocorrências de edifícios com número elevado de pavimentos, variando entre 24 e 42 dentre as soluções selecionadas.

Também porque as soluções priorizaram o número reduzido de gabarito máximo, as opções escolhidas não apresentam os melhores resultados para sombreamento em períodos quentes entre os otimizados, apenas valores medianos dentre os encontrados. A alta densidade também afetou o percentual médio de visão de pôr do sol que obteve um valor máximo de $65 \%$ de ocorrência anuais nas soluções selecionadas. Os melhores valores deste indicador também estão associados a maior rugosidade. Ou seja, alta rugosidade produziu o menor impacto visual. Dentre as soluções selecionadas, escolheuse a que atendia melhor aos objetivos da proposta, a solução otimizada 3. Esta apresentou um walkscore médio superior a $90 \%$, um bom atendimento ao valor de exposição de radiação em períodos quentes, exposição mínima das fachadas ao sol e visão do pôr do sol. 


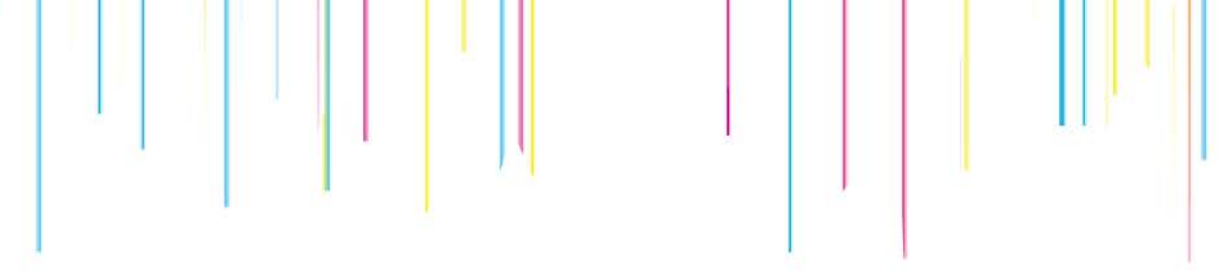

Tabela 3. Resultados obtidos em solução selecionadas na otimização.

\begin{tabular}{cccccc} 
SOLUÇÕES & $\begin{array}{c}\text { Walkscore } \\
\mathbf{( 0 - 1 0 0 )}\end{array}$ & $\begin{array}{c}\text { Horas de vista } \\
\text { do pôr do sol } \\
\text { no ano (\%) }\end{array}$ & $\begin{array}{c}\text { Número } \\
\text { máximo de } \\
\text { gabarito }\end{array}$ & $\begin{array}{c}\text { Área com 20\% de } \\
\text { horas de sol (m²) }\end{array}$ & $\begin{array}{c}\text { Total de radiação em } \\
\text { períodos quentes (Kw) }\end{array}$ \\
\hline 1 & 89,15 & 59,63 & 24 & 639505 & $3,3549 \mathrm{e}+7$ \\
\hline 2 & 89,23 & 59,61 & 26 & 601794 & $3,3477 \mathrm{e}+7$ \\
\hline $\mathbf{3}$ & $\mathbf{9 0 , 7 9}$ & $\mathbf{6 0 , 5 4}$ & $\mathbf{2 8}$ & $\mathbf{6 3 8 9 0 0}$ & $3,3549 \mathrm{e}+7$ \\
\hline 4 & 93,99 & 59,45 & 30 & 700033 & $3,4320 \mathrm{e}+7$ \\
\hline 5 & 89,93 & 60,62 & 32 & 647709 & $3,3559 \mathrm{e}+7$ \\
\hline 6 & 93,56 & 60,3 & 35 & 636723 & $3,2462 \mathrm{e}+7$ \\
\hline 7 & 90,91 & 59,51 & 37 & 636048 & $3,0063 \mathrm{e}+7$ \\
\hline
\end{tabular}

Fonte: Autores, 2021

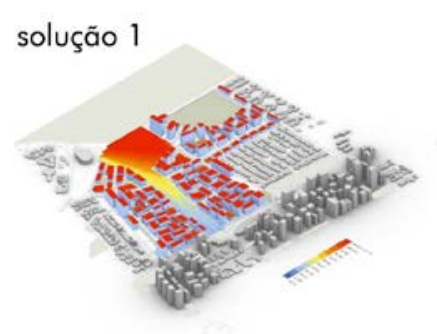

solução 4

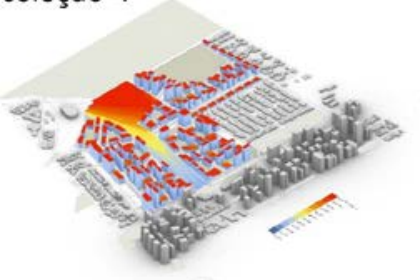

solução 2

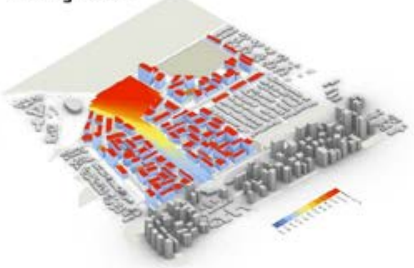

solução 6

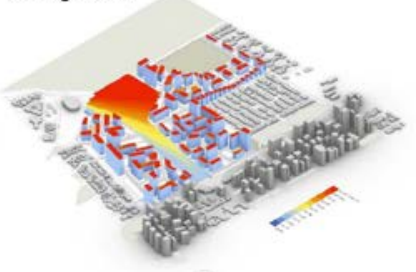

solução 3

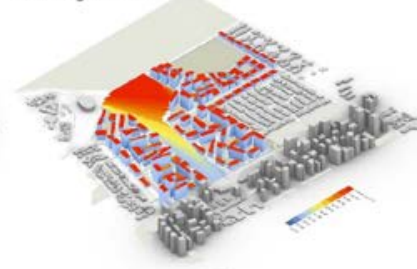

solução 8

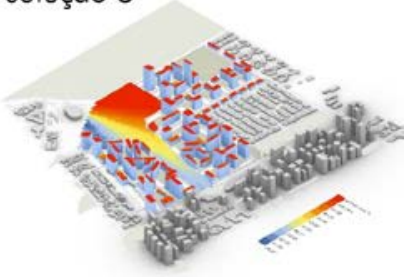

Figura 4. Seis soluções selecionadas na otimização com base no menor gabarito. As imagens mostram o resultado visual para os percentuais de visão do pôr do sol no parque linear e exposição a radiação em períodos quentes nos edifícios. Fonte: Autores, 2021

\section{Discussão}

As funções relevantes foram otimizadas em função de indicadores determinantes para o projeto urbano a partir do modelo paramétrico. A execução do algoritmo genético de otimização multicritério obteve centenas de soluções ótimas definidas na fronteira de pareto que permitem discussões fundamentadas a equipe de projeto baseadas em desempenho. A equipe pode experimentar diferentes combinações a partir dos resultados e estabelecer uma decisão a partir de sua sensibilidade e não apenas baseados em subjetividade.

$O$ projeto baseado em desempenho possui um grande potencial para o planejamento urbano, pois promove a interação integrada associando o poder 


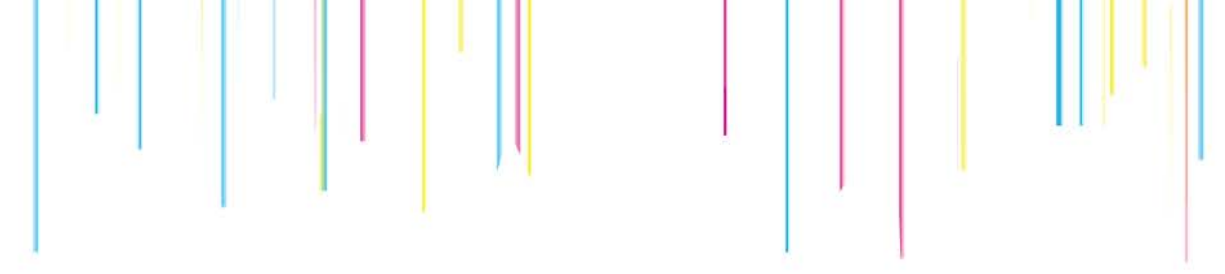

computacional à experiência do profissional. No entanto, uma limitação do experimento foi o processamento, apesar do tempo por ciclo considerado rápido pelos autores. Uma vez que, não foi utilizado processamento em paralelo, ou processamento terceirizado, como na nuvem, gerações adicionais poderiam ter apresentado soluções melhores que as apresentadas no artigo.

As ferramentas de parametrização e otimização não apenas oferecem soluções objetivas, como também permitem que o urbanista vislumbre novas combinações de soluções com mais eficiência. As ferramentas algorítmicas não substituem o agente humano, mas o potencializam.

\section{Referências}

Aish, R. (2011). DesignScript: Origins , Explanation , Illustration. Computational design modelling, [s. I.], p. 1-8.

Anton, I.; Tănase, D. (2016). Informed Geometries. Parametric Modelling and Energy Analysis in Early Stages of Design. Energy Procedia, v. 85, n., p. 9-16

$\mathrm{A}+\mathrm{T}$, Research Group. (n.d.). 50 Urban Blocks2. $A+T$ architecture publishers.

Carone, M. (2016). Computação evolutiva e as cidades do futuro. Instituto de Pesquisa e Inovação em Urbanismo.

Cichocka, J. (2015). Generative design optimization in urban planning: walkabilityoptimized city concept , Architectus, 10, pp. 119-128. doi: 10.5277/arc150110

Dennemark, M. (2018). Divide by Area. Grasshopper snippets for Urban Design and Analysis. DeCodinSpaces Toolbox for Grasshopper.

Duany, A., Steuteville, R. (2021). Defining the 15 minutes city. Congress for the New Urbanism.

Evelin, S.; Jason G., David. (2014). Designing-in performance: A framework for evolutionary energy performance feedback in early stage design. Automation in Construction, v. 38, p. 59-73.

Farr, D. (2013). Urbanismo sustentável: desenho urbano com a natureza. Bookman.

Grobman, Y.J.; Neuman, E. (2008). Performalism: Form and performance in digital architecture Tel Aviv Museum of Art.

Instituto Brasileiro de Geografia e Estatística [IBGE]. (2017). Censo Brasileiro de 2010. Rio de Janeiro: IBGE.

Instituto de Pesquisa, Administração e Planejamento [IPPLAN]. (2014). Atlas da pesquisa origem e destino: panorama da mobilidade em São José dos Campos. São Carlos: Cubo.

Kirimtat, A. et al. (2019). Multi-objective energy and daylight optimization of amorphous shading devices in buildings. Solar Energy, v. 185, n. April, p. 100-111.

Kolarevic, B. (2004). Back to the Future: Performative Architecture. International Journal of Architectural Computing, v. 2, n. 1, p. 43-50.

Kolarevic, B.; Malkawi, A. (2005). Performative Architecture: Beyond Instrumentality 


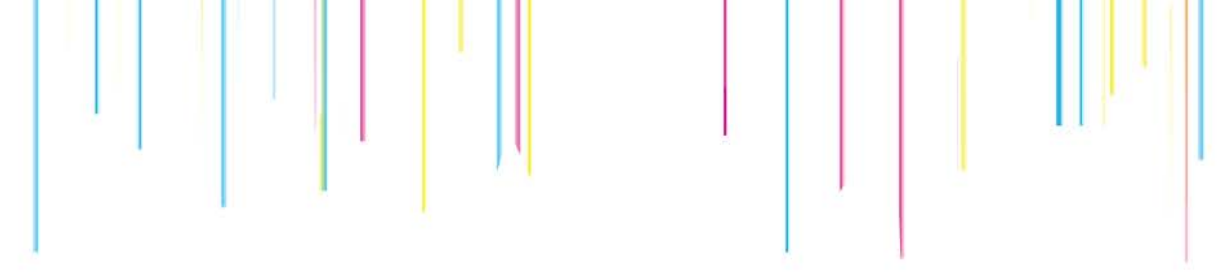

Leite, C.; Awad, J. d. C. M. (2012). Cidades sustentáveis, cidades inteligentes: desenvolvimento sustentável num planeta urbano. Porto Alegre: Bookman.

Lima, F. (2019). Métricas Urbanas: Abordagens paramétricas no planejamento de bairros e cidades sustentáveis. ProBooks.

Lima, F.; Kos, J. R.; Montenegro, N. (2016). Otimização multi-objetivo e desenvolvimento orientado pelo transporte: algoritmos evolutivos em estratégias de planejamento urbano. Blucher Design Proceedings, v. 3, n. 1, p. 601-608.

Lima, F.; Costa, F. R.; Rosa, A. (2020). Lógica algorítmica-paramétrica e urbanismo: uma revisão teórica e de modelos computacionais para projetos urbanos. Gestão \& Tecnologia De Projetos, v. 15, n. 2, p. 84-97.

Lima, F.; Brown, N.; Duarte, J. (2021). Urban Design Optimization: Generative Approaches towards urban fabrics with improved transit accessibility and walkability. 26th International. CAADRIA, Volume 2, 719-728.

Lynch, K. (2011). A imagem da cidade. (3 $3^{\mathrm{a}}$ ed.) São Paulo: Editora WMF Martins Fontes.

Macedo, A. C. (2007). A Carta do Novo Urbanismo norte-americano. Arquitextos, v. 07 (n. 082.03)

Moura, E. R. de. (2006). Sistema de circulação e de transporte e o espaço urbano na cidade de São José dos Campos: um estudo multitemporal. Dissertação (Mestrado) — Universidade do Vale do Paraíba.

Nguyen, A. T.; Reiter, S.; Rigo, P. (2014). A review on simulation-based optimization methods applied to building performance analysis. Applied Energy, [s. I.], v. 113, p. 1043-1058.

Oxman, R. (2008) Performance-based design: current practices and research issues. International journal of architectural computing, SAGE Publications Sage UK: London, England, v. 6, n. 1, p. 1-17.

Oxman, R. (2017). Thinking difference: Theories and models of parametric design thinking. Design Studies, [s. I.], v. 52, p. 4-39.

Prefeitura de São José dos Campos. (2018). Plano Diretor de Desenvolvimento Integrado [PDDI]. Acesso: 30 de jun, 2021, em http://planodiretor.sjc.sp.gov.br/home

Queiroz, N. (2020). Análise de inclinação e fluxo hídrico. Blog bugtecture, 2020. Acesso em: 07 de jun. de 2021, em https://www.bugtecture.com/post/an\%C3\%A1lise-deterreno-e-fluxo-h\%C3\%ADdrico

Rodriguez, K. D. et al. (2016). Princípios e parâmetros do novo urbanismo em territórios planejados no brasil. Universidade Presbiteriana Mackenzie, São Paulo.

Seade. (2021). Perfil dos Municípios Paulistas [Região Administrativa de São José dos Campos]. Acesso: 22 Set 2021, em https://perfil.seade.gov.br/.

Wortmann, T.; Nannicini, G. (2017). Introduction to Architectural Design Optimization. In: City Networks - Planning for Health and Sustainability. [s.I.] : Springer International Publishing. v. 128

Zhao, S.; de Angelis, E. (2019). Performance-based Generative Architecture Design: A Review on Design Problem Formulation and Software Utilization. Journal of Integrated Design and Process Science, [s. I.], p. 1-22. 